\title{
Formation, prediction and analysis of stationary and stable ball-like flames at ultra-lean and normal-gravity conditions
}

\section{Citation for published version (APA):}

Hernandez Perez, F. E., Oostenrijk, B. H., Shoshin, Y., Oijen, van, J. A., \& Goey, de, L. P. H. (2015). Formation, prediction and analysis of stationary and stable ball-like flames at ultra-lean and normal-gravity conditions. Combustion and Flame, 162(4), 932-943. https://doi.org/10.1016/j.combustflame.2014.09.020

\section{Document license: \\ TAVERNE}

DOI:

10.1016/j.combustflame.2014.09.020

Document status and date:

Published: 01/01/2015

\section{Document Version:}

Publisher's PDF, also known as Version of Record (includes final page, issue and volume numbers)

\section{Please check the document version of this publication:}

- A submitted manuscript is the version of the article upon submission and before peer-review. There can be important differences between the submitted version and the official published version of record. People interested in the research are advised to contact the author for the final version of the publication, or visit the $\mathrm{DOI}$ to the publisher's website.

- The final author version and the galley proof are versions of the publication after peer review.

- The final published version features the final layout of the paper including the volume, issue and page numbers.

Link to publication

\section{General rights}

Copyright and moral rights for the publications made accessible in the public portal are retained by the authors and/or other copyright owners and it is a condition of accessing publications that users recognise and abide by the legal requirements associated with these rights.

- Users may download and print one copy of any publication from the public portal for the purpose of private study or research.

- You may not further distribute the material or use it for any profit-making activity or commercial gain

- You may freely distribute the URL identifying the publication in the public portal.

If the publication is distributed under the terms of Article 25fa of the Dutch Copyright Act, indicated by the "Taverne" license above, please follow below link for the End User Agreement:

www.tue.nl/taverne

Take down policy

If you believe that this document breaches copyright please contact us at:

openaccess@tue.nl

providing details and we will investigate your claim. 


\title{
Formation, prediction and analysis of stationary and stable ball-like flames at ultra-lean and normal-gravity conditions
}

\author{
Francisco E. Hernández-Pérez * , Bart Oostenrijk, Yuriy Shoshin, Jeroen A. van Oijen, Laurentius P.H. de Goey \\ Department of Mechanical Engineering, Eindhoven University of Technology, P.O. Box 513, 5600 MB Eindhoven, The Netherlands
}

\section{A R T I C L E I N F O}

\section{Article history:}

Received 15 July 2014

Received in revised form 14 September 2014

Accepted 23 September 2014

Available online 28 October 2014

\section{Keywords:}

Flame balls

Ultra-lean hydrogen-methane-air

combustion

Soret effect

\begin{abstract}
A B S T R A C T
In this research work, we report on the numerical predictions and analysis of stable, stationary and closed burner-stabilized reacting fronts under terrestrial-gravity conditions for ultra-lean hydrogen-methaneair premixed mixtures with a $40 \%$ hydrogen $\left(\mathrm{H}_{2}\right)$ and $60 \%$ methane $\left(\mathrm{CH}_{4}\right)$ fuel composition, specified on a molar basis. The transition from a cap-like to ball-like flame shape with decreasing inlet equivalence ratio is predicted in agreement with experimental observations. The predicted flames are compared to both flames that were studied in experiments and numerical solutions of perfectly-spherical flame balls in the absence of gravity and convection. The comparison includes flame size, lean limits, and when pertinent, standoff distances, all for two different reaction mechanisms. The absolute molar consumption rates of both $\mathrm{H}_{2}$ and $\mathrm{CH}_{4}$ for the limit flame attain maximum values that are significantly larger than those of the corresponding gravity-free flame ball. The fuel supply mechanism of the normal-gravity limit flame is similar to the fuel supply of flame balls in that it is driven by diffusion even away from the flame front. Heat conduction to the tube wall of the burner and convective heat loss are the dominant forms of heat loss. Furthermore, simulations with inclusion of multicomponent transport and Soret and Dufour effects show that the flame size increases for both flame balls and the burner-stabilized flames. For the latter, a slight modification in the stabilization position is found owing to the intensification of the consumption rates of both $\mathrm{H}_{2}$ and $\mathrm{CH}_{4}$ when these effects are accounted for. In summary, the present work considers a new configuration that allows the study of stable and stationary ball-like flames at ultra-lean and nearlimit conditions, and advances the understanding of such flames via detailed numerical computations.
\end{abstract}

(c) 2014 The Combustion Institute. Published by Elsevier Inc. All rights reserved.

\section{Introduction}

The understanding and modeling of premixed combustion at ultra-lean conditions is relevant not only to the design of combustion devices (e.g., gas turbines and reciprocating engines) that can alleviate green-house gas and pollutant emissions, but also to safety considerations for near-limit flammable mixtures. One type of flame that is able to exist at ultra-lean and near-limit conditions is the so called flame ball.

Flame balls are spherical premixed flames that were theoretically predicted as steady-state solutions of a model for heat and mass transfer in the absence of gravity and convection by Zeldovich [1], who also predicted that adiabatic flame balls are unstable. While this latter prediction is further supported by studies based on activation energy asymptotics [2,3], rendering adiabatic flame balls non-existent, apparent flame balls were observed in drop-tower experiments at micro-gravity conditions for lean hydrogen-air

\footnotetext{
* Corresponding author.

E-mail address: F.E.Hernandez.Perez@tue.nl (F.E. Hernández-Pérez).
}

mixtures [4]. Buckmaster and co-workers [5,6] showed that the radial stability of micro-gravity flame balls is due to heat loss by thermal radiation. Subsequent experiments in micro-gravity environments $[7,8]$ further confirmed the formation of visibly spherical and stable flames for mixtures close to the lean flammability limit and characterized by a Lewis number (ratio of the mixture thermal diffusivity to the mass diffusivity of the deficient reactant), Le, smaller than unity. The mixtures considered in the micro-gravity experiments were $\mathrm{H}_{2}-$ air, $\mathrm{H}_{2}-\mathrm{O}_{2}-\mathrm{CO}_{2}, \mathrm{H}_{2}-\mathrm{O}_{2}-\mathrm{SF}_{6}$, and $\mathrm{CH}_{4}-\mathrm{O}_{2}-\mathrm{SF}_{6}$. A remarkable feature of flame balls is that the reactant supply needed to maintain combustion is provided solely by diffusion. It should also be noted that multiple flame balls have been generated in the micro-gravity experiments and observed for up to $500 \mathrm{~s}$, experiencing low and repulsive drift speeds [8].

Comprehensive one-dimensional simulations of perfectlyspherical flame balls have been conducted with detailed submodels for chemical kinetics, transport, and thermal radiation, considering hydrogen as fuel [9-11]. It has been found that the reaction mechanism significantly affects the prediction of flame ball characteristics, even though the chemical kinetic schemes yield accurate 
predictions of laminar flame speeds away from the extinction limits.

Although steady flame balls were deemed non-existent under the influence of terrestrial-gravity owing to natural convection, the experimental and numerical work on transient, nearly-spherical, ultra-lean flames at normal-gravity of Shoshin and co-workers $[12,13]$ opened the possibility for their existence. In fact, they identified a transition to a ball-like regime for flames of hydrogenmethane-air mixtures propagating in open-end tubes [13]. Recently, steady, stable and closed burner-stabilized reacting fronts resembling flame balls were observed and characterized under terrestrial-gravity and near-limit conditions by Oostenrijk [14] for hydrogen-methane-air mixtures. Of particular note is the fact that the stabilization and steadiness of such reacting fronts in a wellcontrolled environment allow for the detailed investigation of combustion processes and model validation at ultra-lean and near-limit conditions.

In this work, we report on the numerical predictions and analysis of these steady, stable and closed reacting fronts at normalgravity for ultra-lean hydrogen-methane-air mixtures with a $40 \%$ hydrogen $\left(\mathrm{H}_{2}\right)$ and $60 \%$ methane $\left(\mathrm{CH}_{4}\right)$ fuel composition, specified on a molar basis. A transition from a cap-like to ball-like flame shape is predicted and examined as a function of the inlet equivalence ratio, $\phi$, of the premixed mixture. The predicted flames are compared to both flames that were studied in the experiments of Oostenrijk [14] and numerical solutions of perfectly-spherical flame balls in the absence of gravity and convection. The comparison includes flame size, lean limits, and when appropriate, standoff distances, all for two different chemical kinetic schemes. Heat losses via thermal radiation and heat conduction are quantified and their influence on the stabilization of the flames is also discussed. Moreover, simulations with inclusion of Soret and Dufour effects are performed. The implications of including such effects in the predicted flame characteristics are highlighted.

In the following sections the burner configuration and numerical modeling approach are described, and the numerical and experimental results are discussed subsequently.

\section{Burner configuration}

The experimental setup is described in detail in [14]. Individual laminar flames corresponding to premixed $\mathrm{H}_{2}-\mathrm{CH}_{4}$-air mixtures were stabilized in a cylindrical burner, which has a sapphire tube with a $30 \mathrm{~mm}$ height, $13.5 \mathrm{~mm}$ internal diameter and $1 \mathrm{~mm}$ wall thickness. A schematic of the burner is displayed in Fig. 1. The temperature of the tube wall was kept nearly constant by supplying

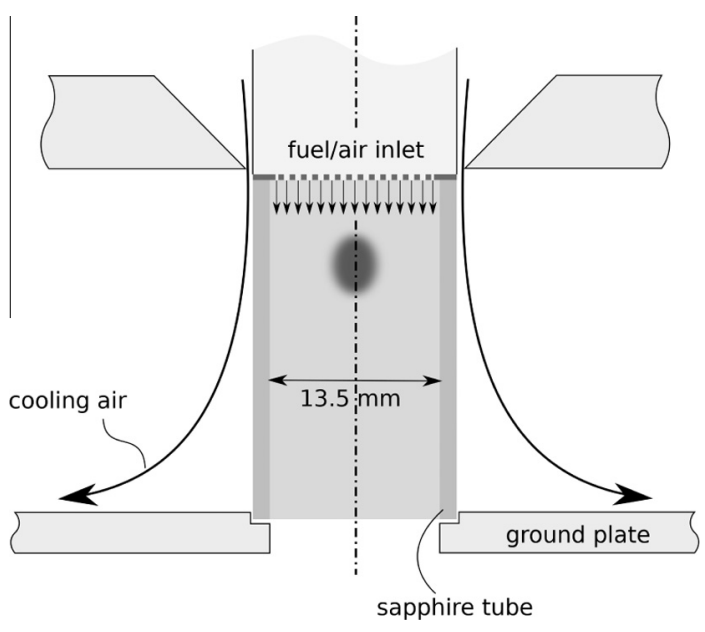

Fig. 1. Schematic of the burner.
Table 1

Summary of main conditions for the cases that have been simulated.

\begin{tabular}{lllll}
\hline Case & Fuel & $\frac{n_{\mathrm{H}_{2}}}{n_{\mathrm{H}_{2}}+n_{\mathrm{CH}_{4}}}$ & $\phi$ & $V_{\text {in }}(\mathrm{m} / \mathrm{s})$ \\
\hline A & $\mathrm{H}_{2}-\mathrm{CH}_{4}$ & 0.4 & 0.4 & 0.072 \\
B & $\mathrm{H}_{2}-\mathrm{CH}_{4}$ & 0.4 & 0.35 & 0.072 \\
C & $\mathrm{H}_{2}-\mathrm{CH}_{4}$ & 0.4 & 0.33 & 0.072 \\
D & $\mathrm{H}_{2}-\mathrm{CH}_{4}$ & 0.4 & 0.325 & 0.072 \\
\hline
\end{tabular}

cooling air through a co-axial slit situated above the burner tube. In effect, the temperature of the external surface of the tube was monitored with a small spot $(2 \mathrm{~mm})$ pyrometer Pyrospot DT 40L. The measured temperature in all the experiments was in the range of $313-323 \mathrm{~K}$ for the entire tube external surface. Due to the high thermal conductivity of sapphire, the difference between the temperature of the external and internal tube surfaces was rather small. According to performed estimations, this difference did not exceed $1 \mathrm{~K}$ in all of the experiments. Moreover, a $3 \mathrm{~mm}$ thick perforated plate having uniformly spaced holes of $0.5 \mathrm{~mm}$ diameter with a $0.7 \mathrm{~mm}$ pitch was mounted above the sapphire tube to supply the premixed mixture with a nearly uniform velocity profile.

The mixture was ignited with a household lighter at an equivalence ratio that was high enough to provide reliable ignition, which was then reduced gradually to the desired value. Ball-like burning structures were formed only when hydrogen was present in the premixed mixture of reactants. The hydrogen molar fraction in the fuel mixture was varied with steps of $20 \%$. It was observed that at a $20 \%$ of hydrogen content in the fuel, ball-like flames could already be formed. The steady flames typically burned for tens of minutes and exhibited no memory on the ignition process. $\mathrm{CH}^{*}$ chemiluminescence of the flames was recorded by an AVT-PIKE F-032b CCD-camera equipped with an interference filter $(430 \mathrm{~nm}$, bandwidth $10 \mathrm{~nm}$ ). The chemiluminescence images show a lineof-sight integrated emission intensity of cylindrically symmetric flames. The in-plane radial emission intensity distribution was recovered by Abel inversion.

Even though reactive mixtures with different hydrogen content and inflow velocities were studied in the experiments, the present research work is limited to mixtures containing $40 \%$ (on a molar basis) hydrogen content in the fuel and having an inflow velocity, $V_{\text {in }}$, of $7.2 \mathrm{~cm} / \mathrm{s}$ at atmospheric pressure. The $40 \% \mathrm{H}_{2}-60 \% \mathrm{CH}_{4}$ fuel mixture was chosen because it contains a significant amount of $\mathrm{CH}_{4}$ and still exhibits stable ball-like structures as the limit flame is approached. The conditions for the cases considered in this study are all summarized in Table 1.

\section{Governing equations}

The flames under study are both laminar and axisymmetric. They are mathematically described by conservation equations for mass, momentum, energy and species in a cylindrical coordinate system. The flow is treated as a continuous, multicomponent, compressible, and thermally-perfect mixture of gases. A Newtonian flow is assumed and the mixture is assumed to obey the ideal gas equation of state. The conservation equations for a reactive mixture of $N$ chemical species evolving in time, $t$, and space, $\vec{x}=(r, \theta, z)$, can be expressed in axisymmetric form as

$\frac{\partial \rho}{\partial t}+\frac{\partial\left(\rho u_{\mathrm{r}}\right)}{\partial r}+\frac{\partial\left(\rho u_{\mathrm{z}}\right)}{\partial z}=-\frac{\rho u_{\mathrm{r}}}{r}$

$\frac{\partial\left(\rho u_{\mathrm{r}}\right)}{\partial t}+\frac{\partial\left(\rho u_{\mathrm{r}}^{2}+p\right)}{\partial r}+\frac{\partial\left(\rho u_{\mathrm{r}} u_{\mathrm{z}}\right)}{\partial z}=\frac{\partial \tau_{\mathrm{rr}}}{\partial r}+\frac{\partial \tau_{\mathrm{rz}}}{\partial z}+\frac{\tau_{\mathrm{rr}}-\tau_{\theta \theta}-\rho u_{\mathrm{r}}^{2}}{r}$,

$\frac{\partial\left(\rho u_{\mathrm{z}}\right)}{\partial t}+\frac{\partial\left(\rho u_{\mathrm{z}} u_{\mathrm{r}}\right)}{\partial r}+\frac{\partial\left(\rho u_{\mathrm{z}}^{2}+p\right)}{\partial z}=\frac{\partial \tau_{\mathrm{rz}}}{\partial r}+\frac{\partial \tau_{\mathrm{zz}}}{\partial z}+\frac{\tau_{\mathrm{rz}}-\rho u_{\mathrm{z}} u_{\mathrm{r}}}{r}+\rho g_{\mathrm{z}}$, 


$$
\begin{aligned}
& \frac{\partial(\rho E)}{\partial t}+\frac{\partial\left[\rho u_{\mathrm{r}}(E+p / \rho)\right]}{\partial r}+\frac{\partial\left[\rho u_{\mathrm{z}}(E+p / \rho)\right]}{\partial z} \\
& =\frac{\partial\left(u_{\mathrm{r}} \tau_{\mathrm{rr}}+u_{\mathrm{z}} \tau_{\mathrm{rz}}-q_{\mathrm{r}}\right)}{\partial r}+\frac{\partial\left(u_{\mathrm{r}} \tau_{\mathrm{rz}}+u_{\mathrm{z}} \tau_{\mathrm{zz}}-q_{\mathrm{z}}\right)}{\partial z} \\
& \quad-\frac{\rho u_{\mathrm{r}}(E+p / \rho)}{r}+\frac{u_{\mathrm{r}} \tau_{\mathrm{rr}}+u_{\mathrm{z}} \tau_{\mathrm{rz}}-q_{\mathrm{r}}}{r}+\rho u_{\mathrm{z}} g_{\mathrm{z}}, \\
& \frac{\partial\left(\rho Y_{\alpha}\right)}{\partial t}+\frac{\partial\left[\rho Y_{\alpha}\left(u_{\mathrm{r}}+V_{\alpha, \mathrm{r}}\right)\right]}{\partial r}+\frac{\partial\left[\rho Y_{\alpha}\left(u_{\mathrm{z}}+V_{\alpha, \mathrm{z}}\right)\right]}{\partial z}=-\frac{\rho Y_{\alpha}\left(u_{\mathrm{r}}+V_{\alpha, \mathrm{r}}\right)}{r}+\dot{\omega}_{\alpha},
\end{aligned}
$$

with $\alpha=1, \ldots, N-1$, and the subscripts $\mathrm{r}, \theta$ and $\mathrm{z}$ referring to components in the radial, angular and axial directions, respectively. In the expressions above, $\rho$ is the mixture density, $\vec{u}$ is the mixture velocity, $p$ is the mixture pressure, $T$ is the mixture temperature, $E$ is the total mixture energy (including chemical energy or heat of formation), $Y_{\alpha}$ is the mass fraction of species $\alpha, \dot{\omega}_{\alpha}$ is the net reaction rate of species $\alpha, \vec{g}$ is the acceleration due to gravity, $\overrightarrow{\vec{\tau}}$ is the viscous stress tensor, $\vec{q}$ is the heat flux vector (energy flux due conduction, energy flux due to diffusion and thermal radiation energy flux), and $\vec{V}_{\alpha}$ is the diffusion velocity of the species $\alpha$.

As a first approximation, Soret (diffusion of species due to a temperature gradient) and Dufour (diffusion of heat due to gradients of species) effects are neglected, and multispecies diffusion is modeled using the first-order Hirschfelder and Curtiss approximation [15]. Later, in Section 5.6, Soret and Dufour effects are accounted for and the full multicomponent diffusion formulation is employed.

\section{Numerical solution procedure}

The set of governing equations (Eqs. (1)-(5)) is solved using a body-fitted, multi-block, adaptive mesh refinement (AMR), finitevolume framework that has been originally developed by Groth and co-researchers [16-21], which has been extended to model the configuration under study. Applications of this framework to reacting flows have included laminar atmospheric $[17,20]$ and high-pressure sooting [19,22] flames, as well as turbulent premixed $[21,23]$ and non-premixed $[18,20]$ flames, among others. Thermodynamic properties, transport properties and species net production/destruction rates are all evaluated utilizing the open-source package CANTERA [24,25].

Chemical kinetics is chiefly described by the GRI-Mech 3.0 reaction mechanism [26]. GRI-Mech 3.0 has been used previously to obtain predictions of laminar flame speeds, profiles of major species and extinction strain rates for hydrogen-methane mixtures, which are in agreement with experimental values [27,28]. Since this research is not focused on prediction of oxides of nitrogen $\left(\mathrm{NO}_{x}\right)$, nitrogen $\left(\mathrm{N}_{2}\right)$ is assumed to be inert resulting in a modified version of the mechanism consisting of 36 species with 219 reactions. Also, to examine the influence of the chemical kinetic scheme on the numerical predictions, additional computations are carried out with a skeletal mechanism consisting of 16 species and 25 reaction steps [29]. This skeletal mechanism was previously employed by Shoshin et al. [13] to simulate ball-like propagating fronts of hydrogen-methane mixtures in open-end tubes, obtaining good qualitative results.

Furthermore, as thermal radiation has been found to play a major role in the stabilization of micro-gravity flame balls, it is incorporated in the modeling via the optically-thin Planck model. The Planck mean absorption coefficient of the mixture is evaluated from the major radiating species $\mathrm{CO}_{2}, \mathrm{H}_{2} \mathrm{O}$ and $\mathrm{CO}$. The individual coefficients are calculated using a statistical narrow-band model and the dataset of Soufiani and Taine [30].

In the simulations, a two-dimensional (2D) rectangular domain having a width of $6.75 \mathrm{~mm}$ and a height of $30 \mathrm{~mm}$ is used (Fig. 2). The axis of symmetry is aligned with the left boundary of the

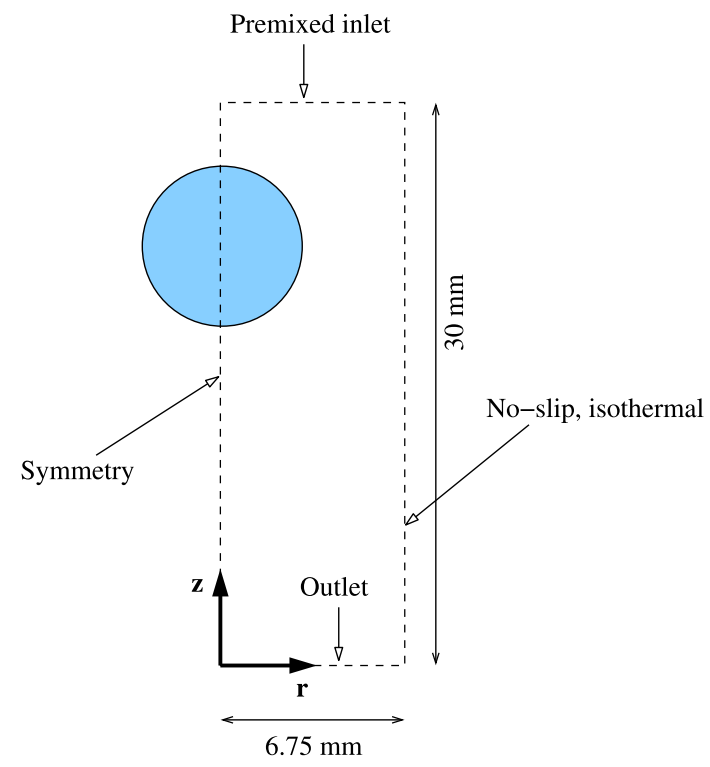

Fig. 2. Schematic of the two-dimensional computational domain and boundary conditions.

domain. The fuel/air premixed mixture is supplied uniformly from the upper boundary, the side tube wall is modeled as a no-slip and isothermal boundary, and the lower boundary is set to a free outflow. The rationale behind the treatment of the tube wall as an isothermal boundary is a modest increase in its temperature $(\Delta T<23 \mathrm{~K})$ that was found in the experiments, with maximum variations in the axial direction of approximately $4 \mathrm{~K}$. It should be noted that, because of preferential diffusion effects, it was needed to account for diffusion of the species across the inflow boundary; otherwise, unrealistic solutions were obtained for inlet equivalence ratios lower than 0.4 .

The transport equations were solved on multi-block quadrilateral meshes employing a second-order spatial discretization. The inviscid flux at each cell face was evaluated using limited linear reconstruction. In particular, the $\mathrm{AUSM}^{+}$-up [31] flux function along with the Venkatakrishnan [32] limiter were utilized. The viscous fluxes were evaluated utilizing a diamond-path reconstruction [33]. Moreover, the low-Mach number preconditioner described by Weiss and Smith [34] and the explicit multi-stage optimally-smoothing time marching scheme of van Leer et al. [35] were used to obtain time-invariant solutions. The computational domain was initially discretized with 4 blocks in the radial direction and 12 blocks in the vertical direction, each one consisting of $8 \times 12$ quadrilateral cells, and subsequently refined during the computations. The steady-state solutions were obtained with up to three levels of refinement, achieving a minimum cell spacing of $52.4 \mu \mathrm{m}$. This level of resolution was found sufficient to resolve the flame front and provide grid-independent solutions.

\section{Results and discussion}

\subsection{Flame shape and overall structure}

In Figs. 3 and 4 the distributions of temperature and heat release for the four cases corresponding to different inlet equivalence ratios are displayed. These solutions were obtained with mixture-averaged transport and the GRI-Mech 3.0 reaction mechanism. It can be seen that a nearly-spherical shape of the reacting front develops as the lean flammability limit is approached. The numerical simulations predict a recirculation zone in the hot core region as the flame adopts the nearly-spherical shape, which is expected to be 


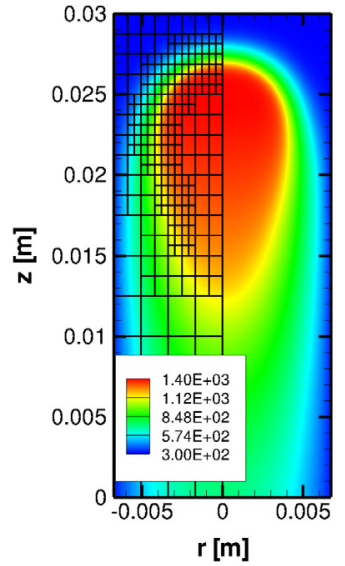

(a) $\phi=0.4$

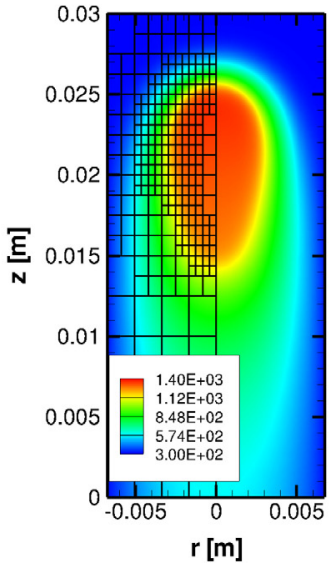

(b) $\phi=0.35$

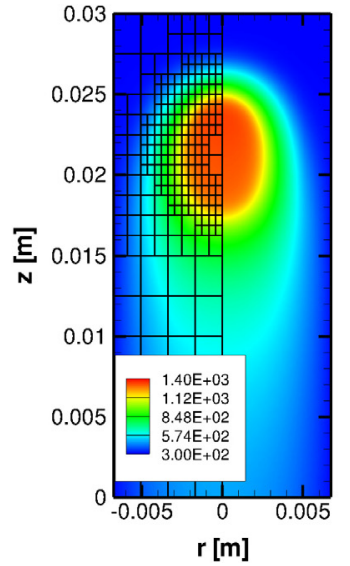

(c) $\phi=0.33$

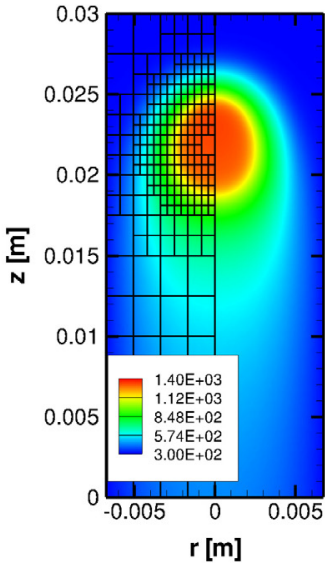

(d) $\phi=0.325$

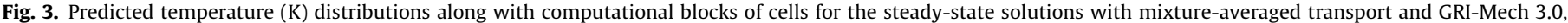
corresponding to the various inlet equivalence ratios $(\phi)$. Each block consists of $8 \times 12$ quadrilateral cells.

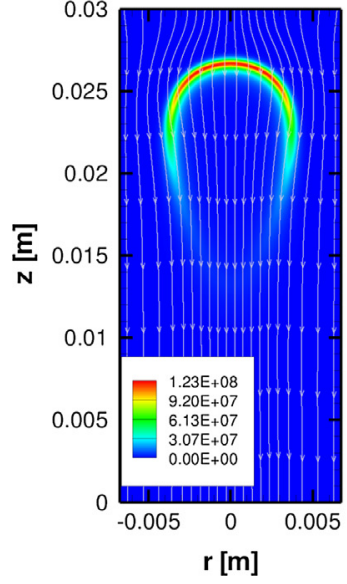

(a) $\phi=0.4$

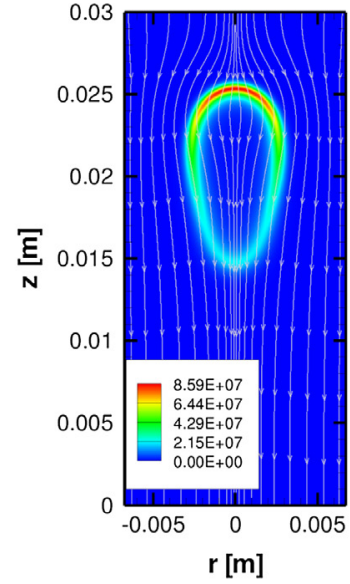

(b) $\phi=0.35$

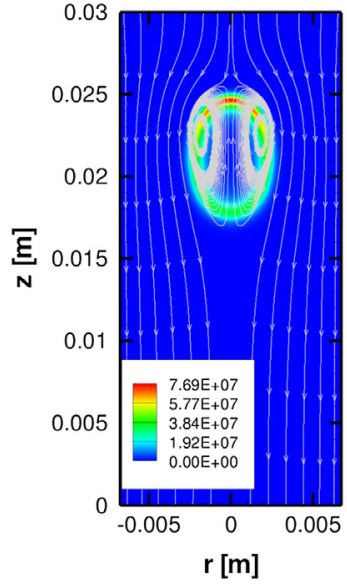

(c) $\phi=0.33$

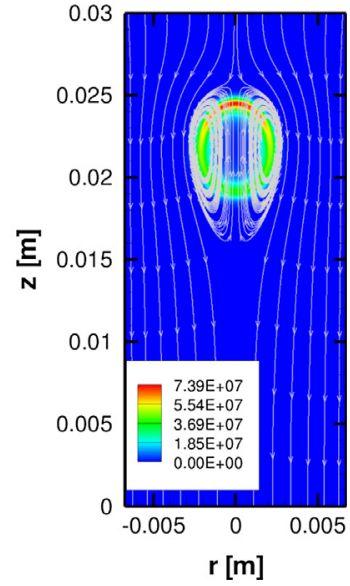

(d) $\phi=0.325$

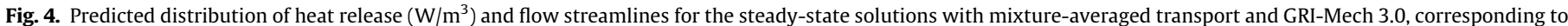
the various inlet equivalence ratios $(\phi)$.

buoyancy-driven as a consequence of the thermal expansion of the fluid in the vicinity of the flame front. For qualitative comparison, $\mathrm{CH}^{*}$ chemiluminescence images of flames studied in the experiments with inlet equivalence ratios ranging from $\phi=0.38$ to $\phi=0.31$ are shown in Fig. 5. It is apparent that the numerical predictions are able to reproduce, at least qualitatively, the transition from a cap-like to a nearly spherical flame. It should be noted that, in terms of flammability, the leanest mixture for which a 2D solution could be obtained corresponds to $\phi=0.325$. However, the limit flame in the experiments corresponds to $\phi \approx 0.31$.

The centerline variations of temperature, heat release, net consumption rates of $\mathrm{CH}_{4}$ and $\mathrm{H}_{2}$, and axial component of velocity are all displayed in Fig. 6. From Fig. 6(a) it can be seen that, regardless of the inlet equivalence ratio, the profiles of temperature exhibit a similar trend, i.e., a rapid increase associated with the reaction zone located a few millimeters away from the inlet, followed by a gradual reduction downstream, which is modest in the hot core region and more pronounced further downstream all the way down to the outlet. Also, as the inlet equivalence ratio decreases, both the length of the hot core region and the maximum value of temperature decrease.

Figure 6(b) reveals the presence of two peaks for the heat release, which become closer to each other as the inlet equivalence ratio decreases. At the leading edge the maximum value of heat release diminishes with decreasing inlet equivalence ratio while
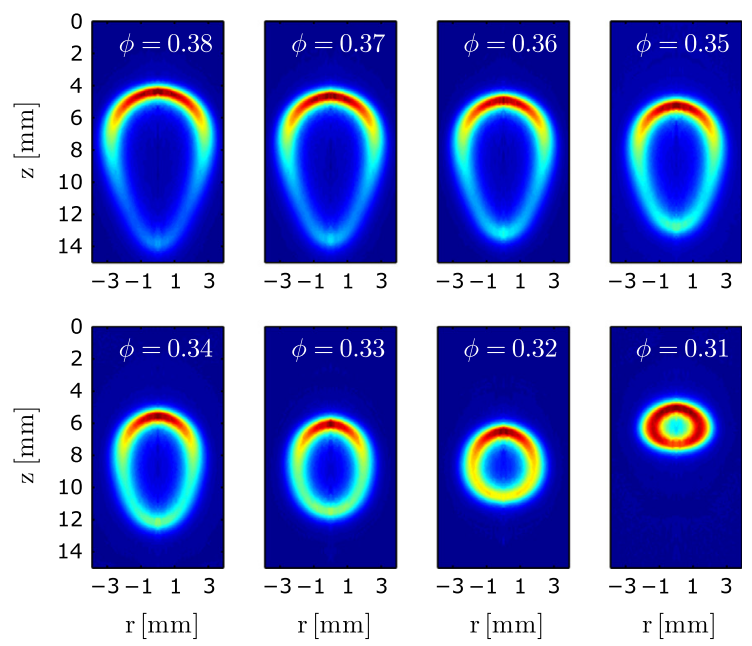

Fig. 5. $\mathrm{CH}^{*}$ chemiluminescence images of the flames studied in the experiments for inlet equivalence ratios ranging from $\phi=0.38$ to $\phi=0.31$. 


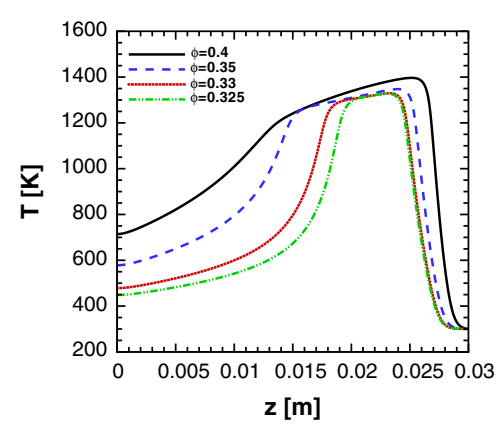

(a)

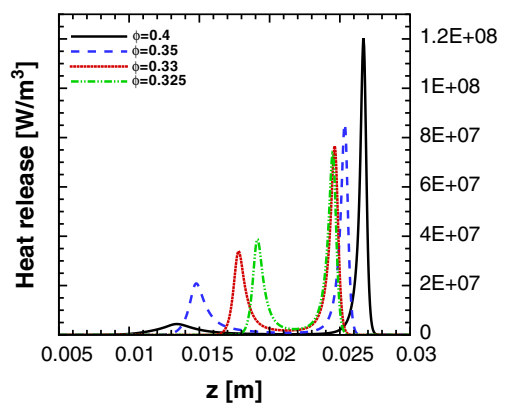

(b)

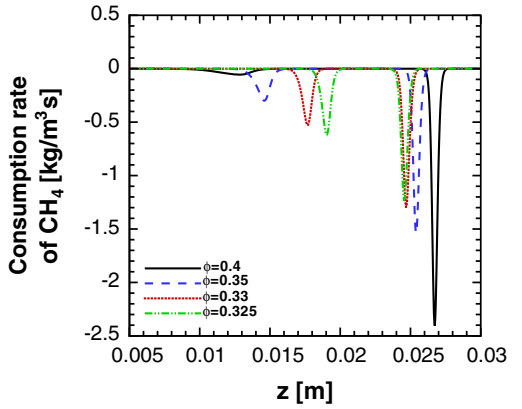

(c)

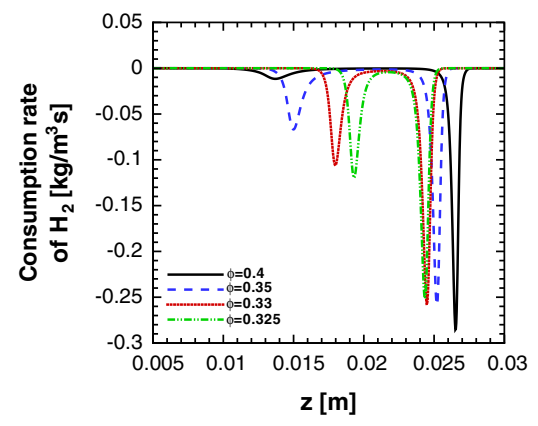

(d)

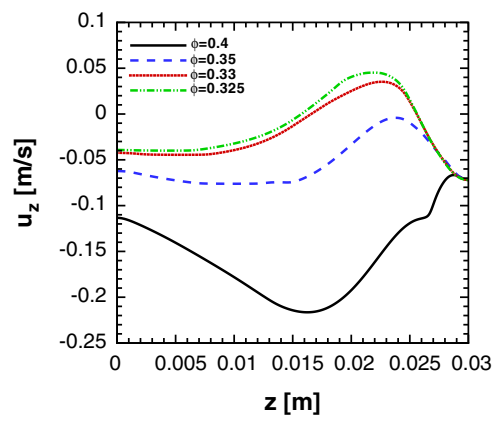

(e)

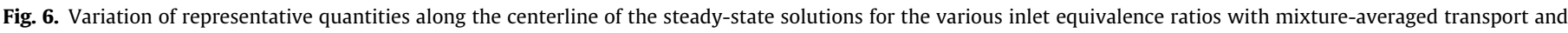
GRI-Mech 3.0. (a) Temperature. (b) Heat release. (c) Net consumption rate of $\mathrm{CH}_{4}$. (d) Net consumption rate of $\mathrm{H}_{2}$. (e) Axial component of velocity.

at the trailing edge the opposite trend is found. The change in shape from a cap-like to ball-like of the flame is thus accompanied by an increment in the heat release at the trailing edge. In terms of the net consumption rates of $\mathrm{CH}_{4}$ (Fig. 6(c)) and $\mathrm{H}_{2}$ (Fig. 6(d)), it can be noted that for all the cases under study both components of the fuel exhibit two peaks in the magnitude of their consumption rates. Likewise the heat release, the peak reduces at the leading edge with diminishing inlet equivalence ratio whereas the opposite trend is observed at the trailing edge. For the trailing edge, the increment in the heat release is caused by the increase in the magnitude of the consumption rates of both fuel components. In addition, the peak values of the absolute rates at the trailing edge represent a larger fraction of their counterparts at the leading edge for $\mathrm{H}_{2}$, as compared to those of $\mathrm{CH}_{4}$.

As for the axial component of velocity (Fig. 6(e)), the solutions for inlet $\phi=0.33$ and $\phi=0.325$ display positive values owing to the presence of the recirculation zones. The distance that separates the two stagnation points is larger for the flame with inlet $\phi=0.325$, indicating a larger extent of the recirculation zones for this case. For the conditions under consideration, a decrease in the inlet equivalence ratio may lead to remarkable changes in the flow pattern and larger residence times.

On the whole, the centerline variations of temperature, heat release and axial component of velocity are qualitatively similar to those reported by Shoshin et al. [13] for rising flame fronts in tubes.

\subsection{Flame size and standoff distance}

To further compare the numerical solutions with the experiments, the half-height and half-width of the flames from the 2D simulations and measurements are plotted in Fig. 7 as a function of the inlet equivalence ratio. Also, the predicted radii for perfectly-spherical flame balls in the absence of gravity and convection (one-dimensional model) are included in Fig. 7. The one-dimensional (1D) flame ball solutions were computed with
CHEM1D [36] using a computational setup similar to the one described in Refs. $[9,11]$. A domain sufficiently long along with an adaptively-refined grid consisting of at least 400 points were utilized to obtain reliable solutions. The characteristic lengths from the numerical solutions are all based on peak values of the heat release, whereas the experimental ones are based on $\mathrm{CH}^{*}$ chemiluminescence.

Overall, the predictions from 2D simulations exhibit a larger size as compared to their experimental counterparts. While the differences are more pronounced for the height, the width displays better agreement with the measurements and more so as the flammability limit is approached. In the case of the experimental flames, the height and width tend to converge to the same value

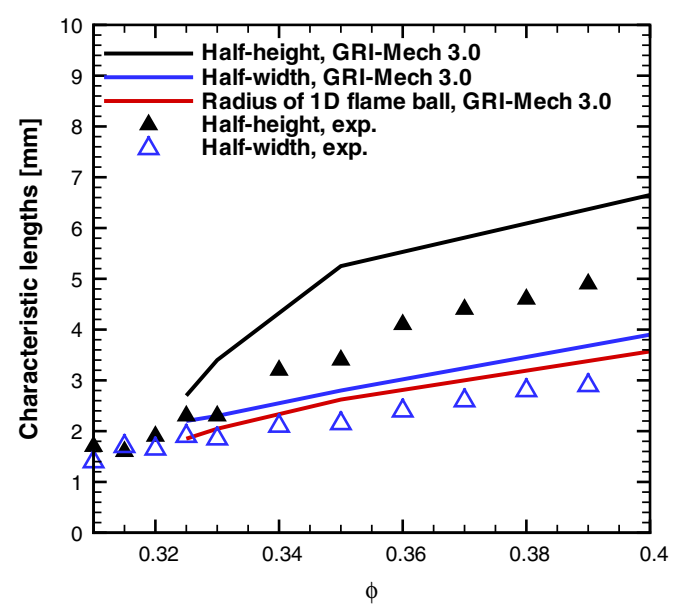

Fig. 7. Half-heights and half-widths from the two-dimensional numerical predictions and chemiluminescence recordings as a function of inlet equivalence ratio. The radii of perfectly-spherical flame balls from one-dimensional simulations are also plotted for comparison. 


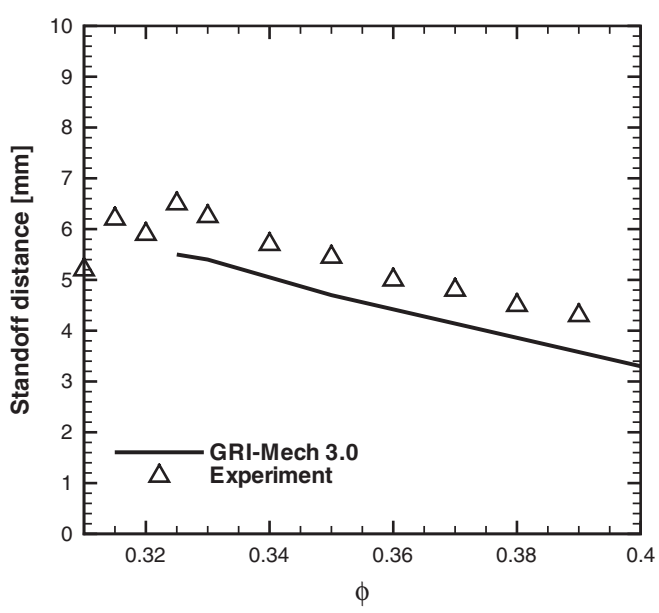

Fig. 8. Standoff distances from the two-dimensional numerical predictions and chemiluminescence recordings as a function of inlet equivalence ratio.

near the flammability limit. In both the 2D predictions and the measurements the height and width of the flame diminish as the inlet equivalence ratio is reduced. The comparison with the $1 \mathrm{D}$ solutions for the gravity-free flame balls reveals that the flame ball radii are comparable with the half-widths of the normal-gravity flames under study.

Another quantity of interest to compare is the standoff distance, which is the distance between the inlet and the leading edge of the flame front along the centerline. To determine the standoff distances, the peaks of heat release are used for the numerical results and those of $\mathrm{CH}^{*}$ chemiluminescence for the experimental ones. From Fig. 8 it is clear that the predicted standoff distances qualitatively agree well with their experimental counterparts. As the inlet equivalence ratio is decreased in the range 0.4-0.325, the standoff distance increases linearly. However, this trend is no longer observed for conditions very close to the limit flame of the experiments $(\phi \approx 0.31)$. The standoff distance for the limit flame decreases instead.

\subsection{Fuel supply analysis}

For a flame ball in the absence of gravity, the fuel is fed to the flame front exclusively by diffusion since there is no convection. However, for the flames at terrestrial-gravity conditions both convection and diffusion are present. The fuel supply mechanisms of the closed flames are further discussed here.

In Fig. 9 the velocity flow field (left) together with the flux lines of $\mathrm{CH}_{4}$ (center) and $\mathrm{H}_{2}$ (right) are shown for the flame with inlet $\phi=0.325$. The flow streamlines (left) and the lines that follow the flux of $\mathrm{CH}_{4}$ (center) and $\mathrm{H}_{2}$ (right) are drawn in blue. Note that only the lines that arrive at the flame front (thick black line) are shown for the $\mathrm{CH}_{4}$ and $\mathrm{H}_{2}$ fluxes. The background color represents the heat release.

The flux lines show that the fluxes of both $\mathrm{CH}_{4}$ and $\mathrm{H}_{2}$ are directed towards the flame front and reach it almost perpendicularly, whereas convection (left plot) is mainly directed downwards outside of both the reaction zone and hot core region. This indicates that diffusion significantly dominates convection even away from the thin reaction zone for both the two components of the fuel, which is an interesting feature of this flame. Nevertheless, a clear difference between the $\mathrm{H}_{2}$ and $\mathrm{CH}_{4}$ fluxes can be observed: the $\mathrm{H}_{2}$ flux lines that arrive at the flame front originate from a wider region at the inflow than those of $\mathrm{CH}_{4}$. This can be attributed to the high diffusivity of $\mathrm{H}_{2}$. Hydrogen more easily diffuses across the flow streamlines, while methane has a larger tendency to follow them.

In Fig. 10 the net molar consumption rates of $\mathrm{H}_{2}$ and $\mathrm{CH}_{4}$ at the centerline of the 2D solution for inlet $\phi=0.325$ are compared with those associated with the corresponding 1D flame ball solution. The maximum absolute values of the molar consumption rates for both $\mathrm{H}_{2}$ and $\mathrm{CH}_{4}$ are considerably larger in the 2D flame (factors of 1.767 and 2.539 for $\mathrm{H}_{2}$ and $\mathrm{CH}_{4}$, respectively) and the rates are not symmetric. Both $\mathrm{H}_{2}$ and $\mathrm{CH}_{4}$ are consumed more vigorously at the leading edge, with peak magnitudes nearly twice as large as those at the trailing edge ( 2.1 for $\mathrm{H}_{2}$ and 1.99 for $\mathrm{CH}_{4}$ ). The difference in the consumption rates between the 1D flame ball and the $2 \mathrm{D}$ flame is due to the fuel supply via both diffusion and convection in the latter case.
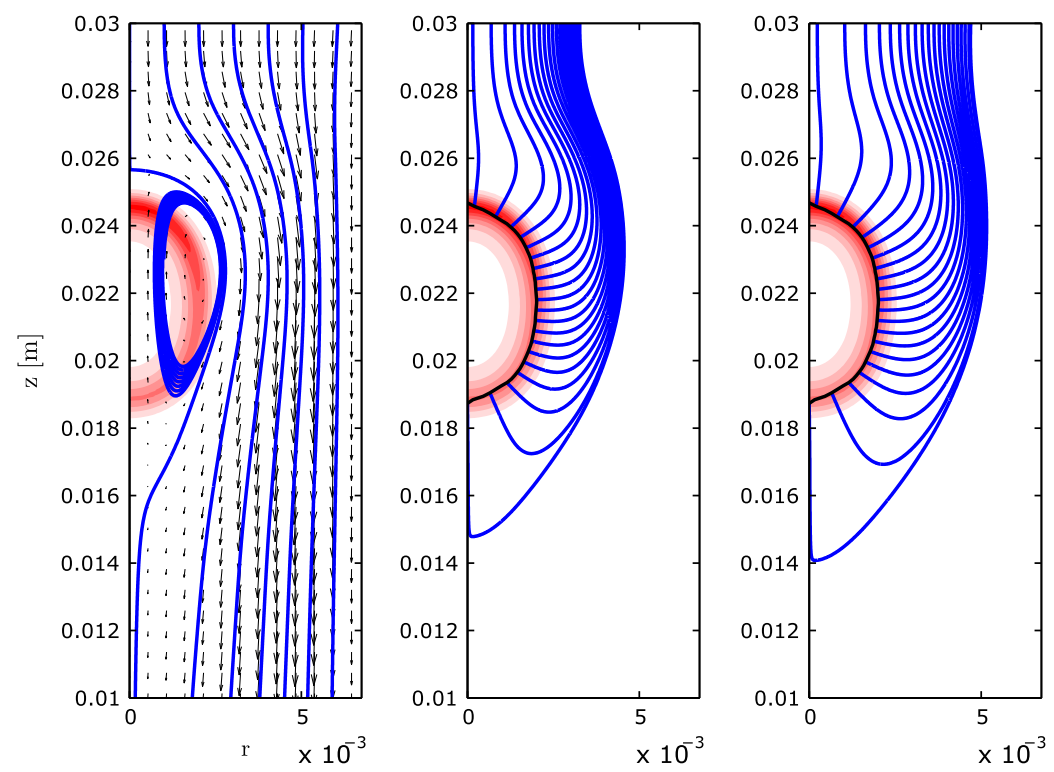

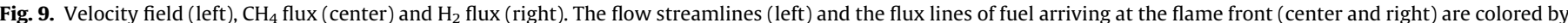

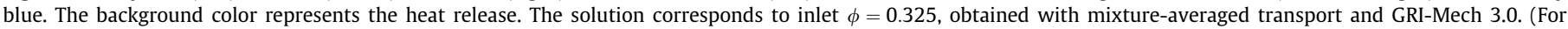
interpretation of the references to color in this figure legend, the reader is referred to the web version of this article.) 


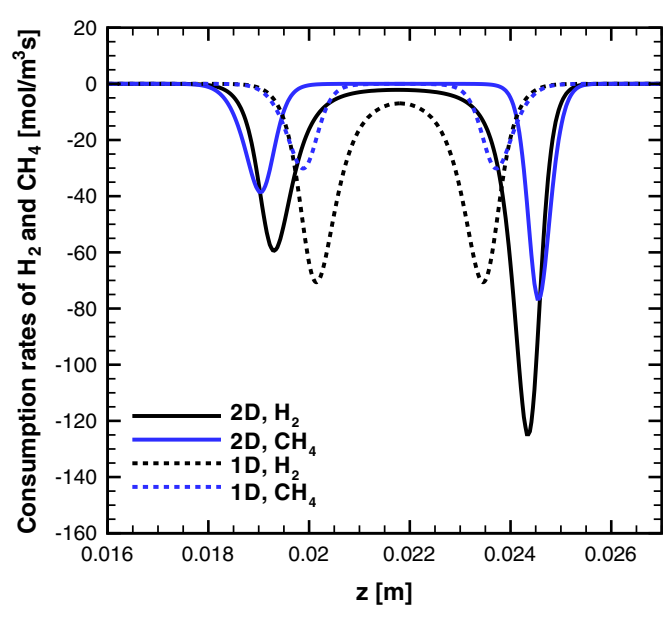

Fig. 10. Net molar consumption rates of $\mathrm{H}_{2}$ and $\mathrm{CH}_{4}$ along the centerline of the leanest two-dimensional solution (inlet $\phi=0.325$ ) and their counterparts for a one-dimensional flame ball. Solutions obtained with mixture-averaged transport and GRI-Mech 3.0.

\subsection{Heat loss}

It has been proven that the gravity-free flame ball is stabilized by means of thermal radiation [5,6]. In the $1 \mathrm{D}$ simulations it is found that the heat release, integrated over the entire flame front, equals the power that is lost by radiation. To quantify heat losses for the burner-stabilized flames and examine their influence on the flame stabilization, the heat release, conductive heat flux to the tube wall and radiative heat loss have all been integrated from the 2D numerical solutions. The results are summarized in Table 2 . The integrated heat release is about $10.29 \mathrm{~W}$ for $\phi=0.4$ and $2.9 \mathrm{~W}$ for $\phi=0.325$. The heat conduction of the fluid to the tube wall is predicted to remain between 61 and 66 percent of the integrated heat release for the flames studied. The calculated integrated heat loss by radiation is approximately $4.7 \%$ of the integrated heat release for $\phi=0.4$ and $2.8 \%$ for $\phi=0.325$. The rest of the released heat is transported out through the outlet.

The heat loss to the tube wall is relatively large and thus has a higher influence on the flame stabilization, as compared to radiation. Due to its low temperature, the tube inner surface prevents the flame from drifting off-center and stabilizes the flame in the horizontal direction. Note that besides contributing to the stability of the flame by heat loss, the tube wall may contribute to stabilize the flame by affecting the flux of reactants to the reaction front. In this regard, the stabilization mechanism is likely to be similar to the one theoretically considered by Buckmaster and Joulin [37] for the case of a gravity-free flame ball stabilized near a flat cold wall. The steady response of such flame ball, based on activation energy asymptotics, indicates that an increase (decrease) of the flame ball size would reduce (augment) the reaction temperature as a consequence of an increase (decrease) in the heat loss to the wall and a decrease (increase) in the reactant mass fraction around the flame.

\section{Table 2}

Integrated heat release $\left(Q_{\text {rel }}\right)$, conductive heat loss to the tube wall $\left(Q_{\text {cond }}\right)$ and radiative heat loss $\left(Q_{\mathrm{rad}}\right)$ from the two-dimensional computations with different inlet equivalence ratios $(\phi)$. The solutions were obtained with mixture-averaged transport and GRI-Mech 3.0.

\begin{tabular}{llll}
\hline Case & $\mathrm{Q}_{\text {rel }}(\mathrm{W})$ & $\mathrm{Q}_{\text {cond }}(\mathrm{W})$ & $\mathrm{Q}_{\text {rad }}(\mathrm{W})$ \\
\hline $\mathrm{A}$ & 10.29 & 6.79 & 0.48 \\
$\mathrm{~B}$ & 5.6 & 3.44 & 0.21 \\
C & 3.5 & 2.17 & 0.11 \\
$\mathrm{D}$ & 2.9 & 1.8 & 0.08 \\
\hline
\end{tabular}

\subsection{Reaction mechanism influence}

Previous computational studies of zero-gravity flame balls have demonstrated that the prediction of flame ball characteristics highly depends on the choice of the reaction mechanism [9-11] even if the reaction scheme accurately predicts flame speeds away from the flammability limits. Hence, the sensitivity of the numerical predictions to the chemical kinetic scheme is also considered and examined here. To this end, a set of steady-state solutions for the conditions given in Table 1 is obtained with the skeletal mechanism [29] described earlier in Section 4, consisting of 16 species and 25 reactions. As previously noted, this scheme was utilized before to study closed propagating fronts of hydrogenmethane mixtures in open-end tubes [13] yielding good qualitative results.

First, it is illustrative to assess the predictive capabilities of the two reaction mechanisms for laminar flame speeds of $1 \mathrm{D}$ planar adiabatic flames at atmospheric pressure and having a fresh gas temperature of $300 \mathrm{~K}$. In Fig. 11 predicted laminar flame speeds are compared to the measurements of Hermanns [38] for lean mixtures with a $40 \%$ hydrogen content in the fuel. Both reaction schemes yield similar values of the laminar flame speeds that agree well with the experimental measurements. Nevertheless, it is worthwhile noting that the skeletal mechanism gives slightly larger flame speeds for mixtures with equivalence ratios below $\phi=0.65$. For example, at $\phi=0.4$, which is a mixture of relevance in the $2 \mathrm{D}$ computations, the predicted flame speeds are $2.31 \mathrm{~cm} / \mathrm{s}$ and $2.57 \mathrm{~cm} / \mathrm{s}$ for the GRI-mech 3.0 and skeletal schemes, respectively (i.e., the flame speed obtained with the skeletal mechanism is $11 \%$ larger than the one obtained with GRI-mech 3.0).

Regarding the burner-stabilized flames, the predicted heat release distributions obtained with the skeletal mechanism are compared to those obtained with GRI-Mech 3.0 in Fig. 12. For consistency of the comparison, all the solutions were obtained with mixture-averaged transport. In each subfigure the left part corresponds to the skeletal mechanism and the right part to GRI-Mech 3.0. It can be seen that the skeletal mechanism yields solutions for reacting fronts that differ in shape and stabilize at different standoff distances. In terms of shape, the differences are more pronounced for inlet $\phi=0.33$ and $\phi=0.325$, i.e., as the flammability limit is approached and the flame becomes more spherical.

To compare in more detail the solutions obtained with the two reaction mechanisms, the isotherms associated with the peak heat release at the trailing edge for each $2 \mathrm{D}$ solution are shown in

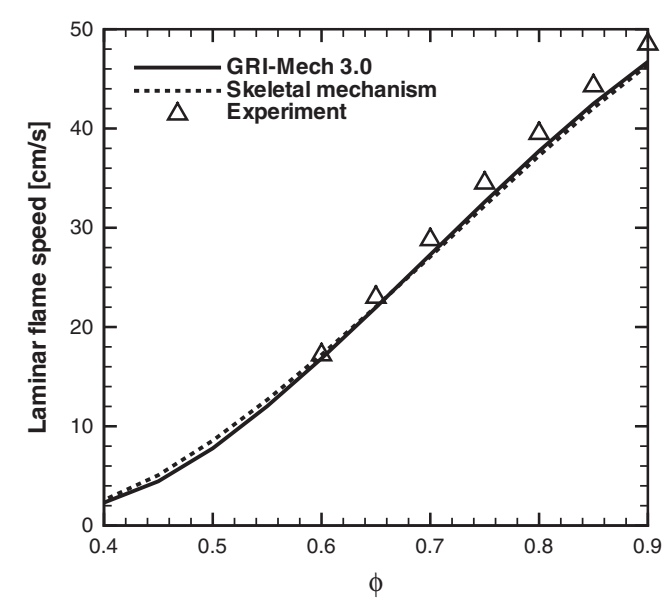

Fig. 11. Comparison of laminar flame speeds for the GRI-Mech 3.0. and skeletal mechanisms with multicomponent transport, against the measurements of Hermanns [38], for mixtures with a $40 \% \mathrm{H}_{2}$ content in the fuel. 


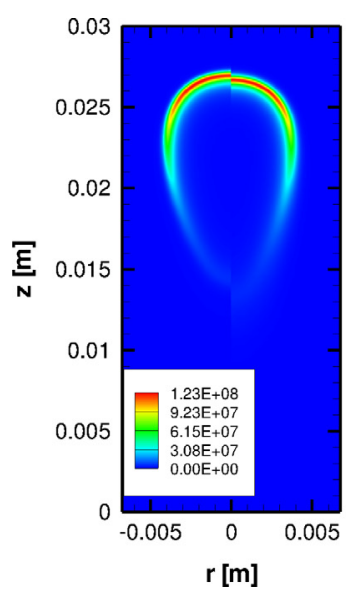

(a) $\phi=0.4$

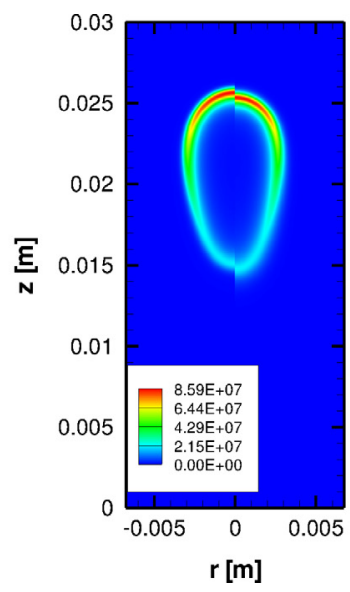

(b) $\phi=0.35$

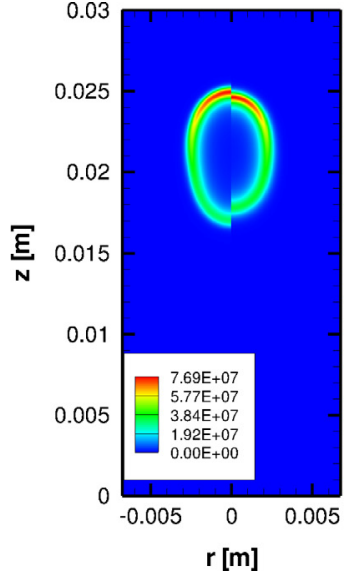

(c) $\phi=0.33$

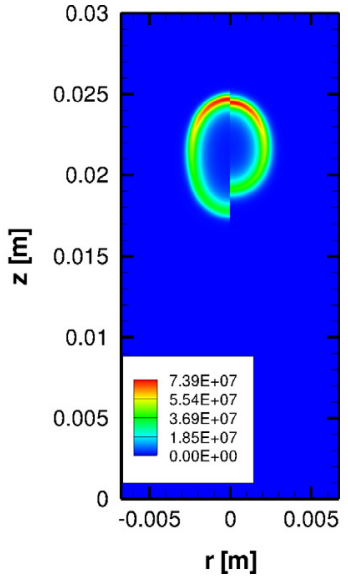

(d) $\phi=0.325$

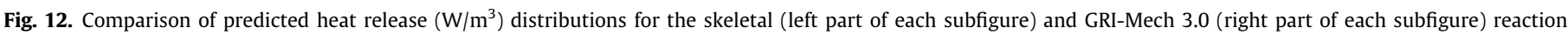
mechanisms with mixture-averaged transport.

Fig. 13, the characteristic lengths of the flames are plotted in Fig. 14, the standoff distances are plotted in Fig. 15, and the variation of representative quantities along the centerline are displayed in Fig. 16.

For the four mixtures studied the skeletal mechanism predicts wider flames as compared to GRI-Mech 3.0 predictions. As for the flame height, the skeletal mechanism yields a shorter flame for inlet $\phi=0.4$, a similar height for $\phi=0.35$, and taller flames for $\phi<0.35$, with respect to GRI-Mech 3.0. Furthermore, when a comparison is made with the experimental measurements, it is evident that GRI-Mech 3.0 gives better predictions of flame width for all the cases studied and better predictions of the height for inlet equivalence ratios lower than 0.35 (Fig. 14).

Also, GRI-Mech 3.0 gives better predictions of standoff distances (Fig. 15). Even though both chemical schemes lead to an underprediction of the standoff distance, the use of the skeletal mechanism results in shorter standoff distances. As in Section 5.2, the predicted radii for perfectly-spherical flame balls in the absence of gravity and convection are also included in Fig. 14 to further compare the two reaction schemes. Larger radii of flame balls are obtained with the skeletal mechanism, which differ more with those obtained with

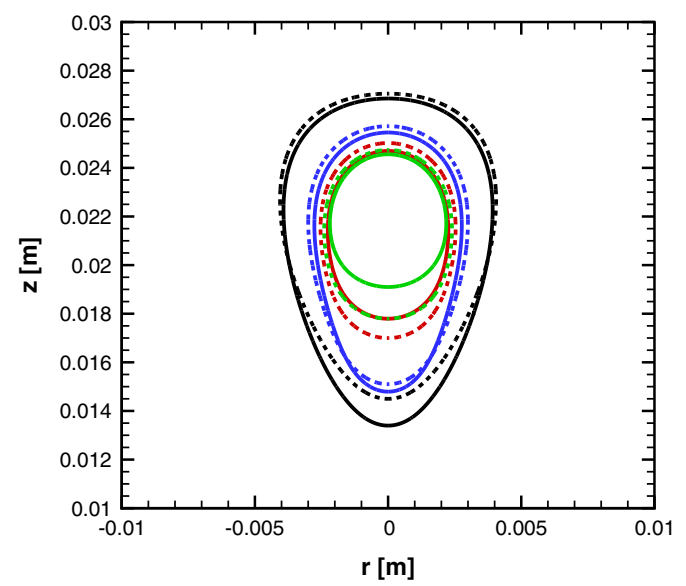

Fig. 13. Isotherms associated with maximum heat release at the trailing edge of the reacting fronts for GRI-Mech 3.0 (solid lines) and skeletal (dashed lines) reaction mechanisms with mixture-averaged transport. Black lines: $\phi=0.4$; blue lines: $\phi=0.35$; red lines: $\phi=0.33$; green lines: $\phi=0.325$. (For interpretation of the references to color in this figure legend, the reader is referred to the web version of this article.)
GRI-Mech 3.0 as the equivalence ratio of the premixed mixture is reduced.

Although there are discernible differences between the predictions of flame size and standoff distance by the two reaction mechanisms, the predictions of flame width and standoff distance are closer to each other, while the differences in flame height are more prominent. By inspecting the variation of representative quantities along the centerline of the two-dimensional solutions, one can elucidate the discrepancies in the predictions of the stabilization position of the leading and trailing edges of the flames, and thereby, those in the flame height. For the leading edge the upstream flow and mixture are more uniform, thus one can expect similar predictions of the stabilization position, with the skeletal mechanism giving shorter standoff distances due to higher burning rates.

As for the trailing edge, owing to the upstream interaction of the flow field, diffusion, chemistry, and heat loss, the composition and temperature of the combustible mixture that feeds the front are modified. In fact, Fig. 16 shows that the mixture downstream of the stabilization point does not only consist of $\mathrm{CH}_{4}, \mathrm{H}_{2}, \mathrm{O}_{2}$, and $\mathrm{N}_{2}$, but other species such as $\mathrm{CO}_{2}, \mathrm{CO}, \mathrm{H}_{2} \mathrm{O}$, which are present in non-negligible amounts. In addition, the mixture there exhibits

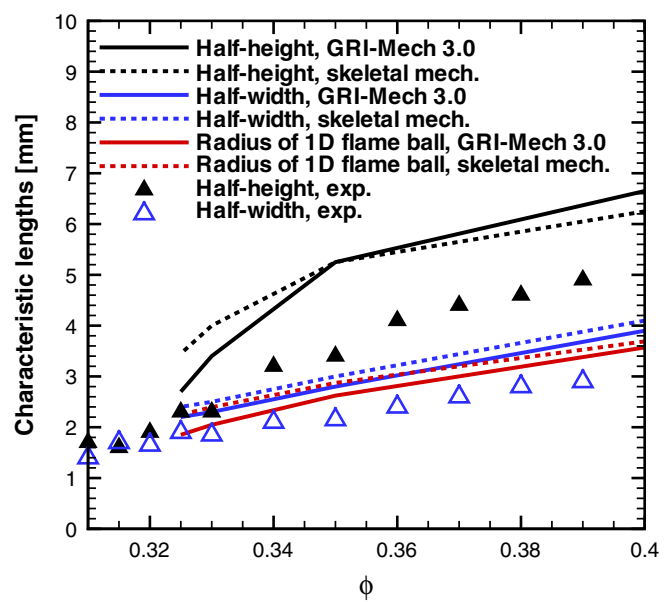

Fig. 14. Half-heights and half-widths from the two-dimensional numerical predictions and chemiluminescence recordings as a function of inlet equivalence ratio. The radii of perfectly-spherical flame balls from one-dimensional simulations are also plotted for comparison. Solutions obtained with mixture-averaged transport and GRI-Mech 3.0 and skeletal mechanisms. 


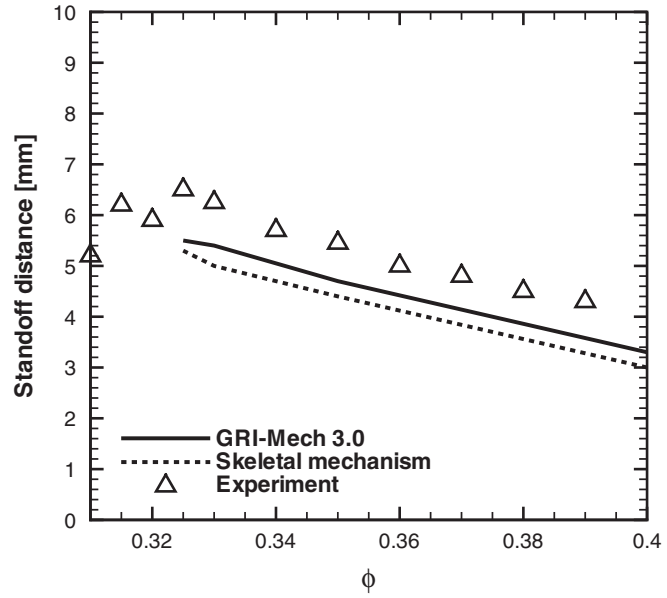

Fig. 15. Predicted standoff distances for GRI-Mech 3.0 (solid lines) and skeletal (dashed lines) reaction mechanisms with mixture-averaged transport.

considerably higher temperatures as compared to the inlet one (e.g., at $z=0.005 \mathrm{~m}$ they are in the range of $482-821 \mathrm{~K}$ ). Hence, the larger discrepancies in the prediction of the stabilization point at the trailing edge can be attributed to the ability of the reaction mechanisms to represent the combustion of mixtures having a more complex composition and considerable degree of preheating.

\subsection{Soret effect}

Thermal diffusion (diffusion of species due to a temperature gradient), also known as the Ludwig-Soret or simply Soret effect, is a second-order transport effect [39]. Under the influence of thermal diffusion, the small and light species are driven towards hotter regions whereas the large and heavy ones are driven towards colder regions. For a wide range of combustion conditions related to $\mathrm{H}_{2}$ and $\mathrm{H}_{2}$-containing fuel blends, the thermal diffusion of $\mathrm{H}_{2}$ and $\mathrm{H}$ has been found to be non-negligible [40-42]. It has been shown that the Soret effect considerably modifies the burning characteristics of two-dimensional, freely-propagating, lean hydrogen-air flames [43]. As the mixtures studied in the present work contain a considerable amount of $\mathrm{H}_{2}$, additional simulations with the inclusion of multicomponent transport and Soret and Dufour (diffusion of heat due to gradients of species) effects are conducted to underscore the influence of second-order diffusion processes on the characteristics of the flames under study.

As pointed out by Sánchez and Williams [40], even though the Dufour effect has been generally found to be negligible, its incorporation in flame calculations is required for a correct entropy production budget [44]. Therefore, it is also incorporated in our computations. Neglecting the terms involving the gradient of pressure in the full multicomponent transport formulation and including thermal radiation in the heat flux vector, the latter can be expressed as

$\vec{q}=\rho \sum_{\alpha=1}^{N} h_{\alpha} Y_{\alpha} \vec{V}_{\alpha}-\lambda \nabla T-\mathcal{R} T \sum_{\alpha=1}^{N} \frac{\mathcal{D}_{\alpha}^{\mathrm{T}} \nabla X_{\alpha}}{\mathcal{M}_{\alpha} X_{\alpha}}-\vec{q}_{\mathrm{rad}}$,

and the diffusion velocity is given by

$\vec{V}_{\alpha}=\frac{1}{X_{\alpha} \mathcal{M}} \sum_{\beta \neq \alpha}^{N} \mathcal{M}_{\beta} \mathcal{D}_{\alpha, \beta} \nabla X_{\beta}-\frac{\mathcal{D}_{\alpha}^{T}}{\rho Y_{\alpha}} \frac{\nabla T}{T}$.

In the equations above $\mathcal{R}$ is the universal gas constant, $\vec{q}_{\text {rad }}$ is the radiative heat flux vector, $\lambda$ is the mixture thermal conductivity, $\mathcal{M}$ is the mixture mean molar mass, and $\mathcal{M}_{\alpha}, X_{\alpha}, h_{\alpha}$, and $\mathcal{D}_{\alpha}^{\mathrm{T}}$ are

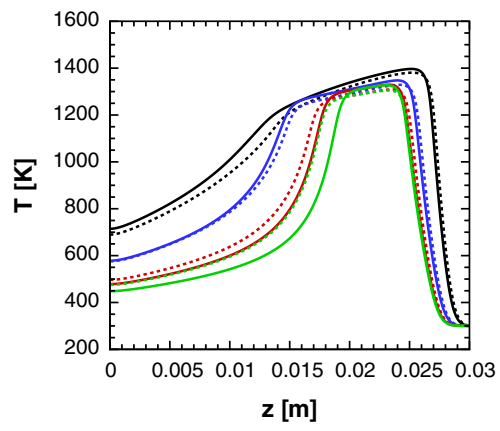

(a)

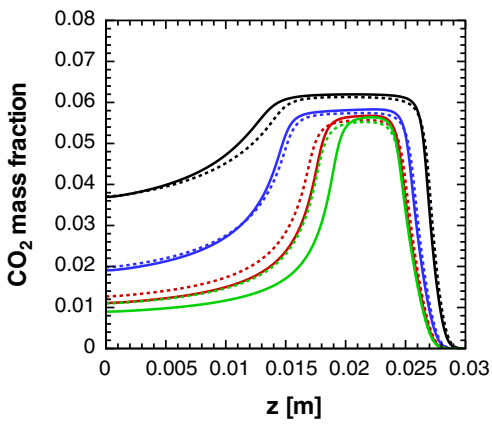

(d)

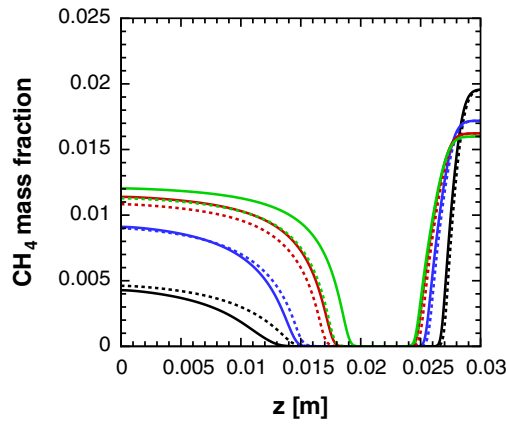

(b)

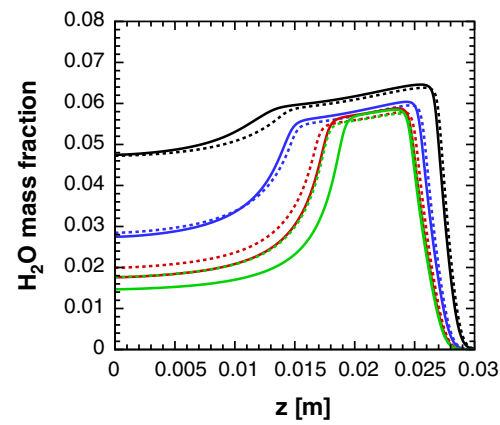

(e)

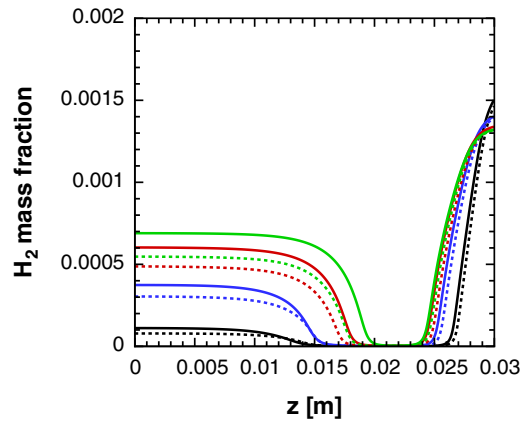

(c)

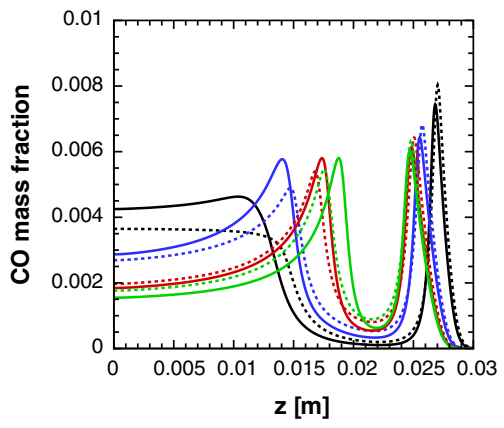

(f)

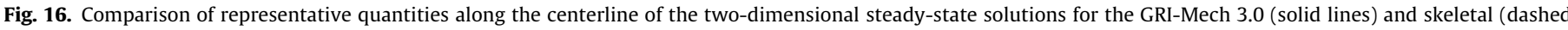

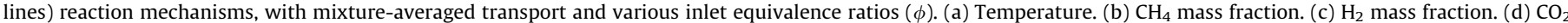

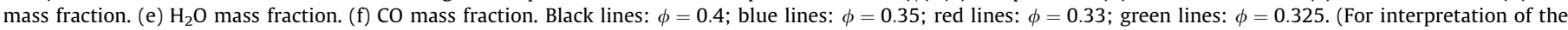
references to color in this figure legend, the reader is referred to the web version of this article.) 


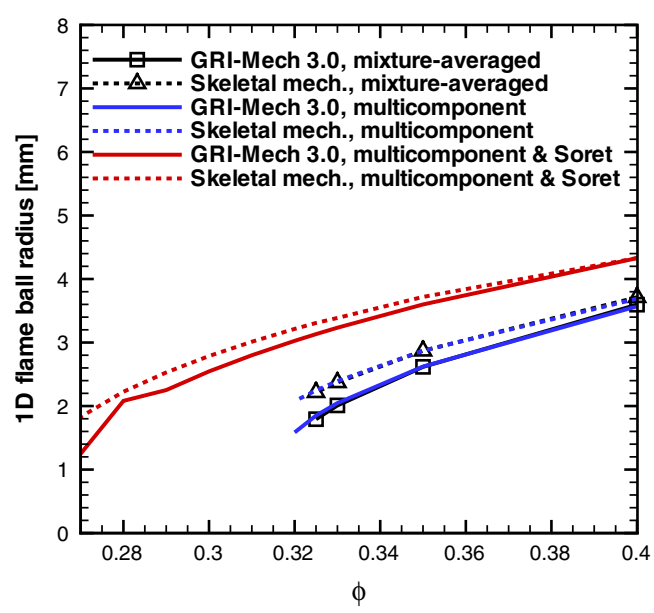

Fig. 17. Influence of transport model and thermal diffusion on the predicted radius for flame balls for GRI-Mech 3.0 and the skeletal mechanism.

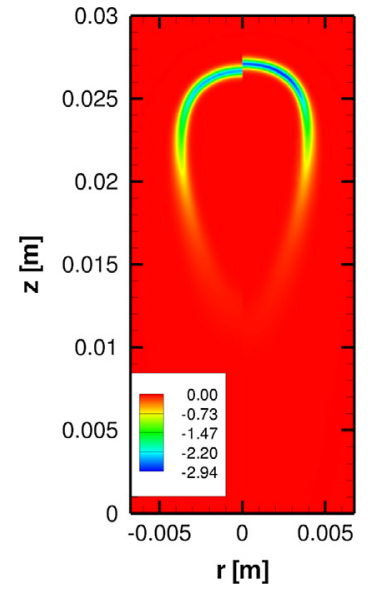

(a) $\dot{\omega}_{\mathrm{CH}_{4}}\left[\mathrm{~kg} \mathrm{~m}^{-3} \mathrm{~s}^{-1}\right]$

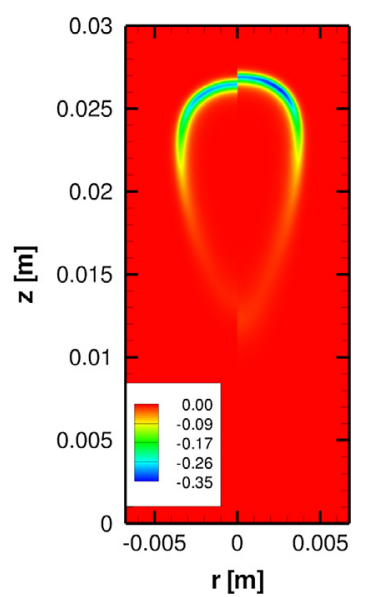

(b) $\dot{\omega}_{\mathrm{H}_{2}}\left[\mathrm{~kg} \mathrm{~m}^{-3} \mathrm{~s}^{-1}\right]$
Fig. 18. Net consumption rates of $\mathrm{CH}_{4}$ and $\mathrm{H}_{2}$ corresponding to steady-state solutions for an inlet equivalence ratio $\phi=0.4$ with mixture-averaged transport (left part of each subfigure), and multicomponent transport with Soret and Dufour effects included (right part of each subfigure). Reaction mechanism: GRI-Mech 3.0.

the molar mass, mole fraction, enthalpy, and thermal diffusion coefficient of species $\alpha$, respectively. The multicomponent diffusion coefficients are denoted by $\mathcal{D}_{\alpha, \beta}$.

The influence of the transport model and thermal diffusion on numerical solutions for perfectly-spherical, gravity-free flame balls is first examined because of the relative simplicity of the 1D model for such flames and the low computational resources associated with their solutions. In Fig. 17 the predicted radii of flame balls with mixture-averaged transport, multicomponent transport, and multicomponent transport with inclusion of Soret and Dufour effects are all displayed. Both reaction mechanisms GRI-Mech 3.0 and the skeletal one are used in this analysis. It can be noted that when Soret and Dufour effects are neglected, both the mixtureaveraged and multicomponent transport models yield very similar predictions of the flame ball radii (black lines with symbols and blue lines, respectively). However, an important difference to remark is the fact that solutions at slightly lower equivalence ratios could be obtained when using multicomponent transport, i.e., a slightly lower lean flammability limit is predicted.

On the other hand, when Soret and Dufour effects are accounted for, both the flame ball radius and the associated lean limit are affected considerably (red lines). Due to the thermal gradient

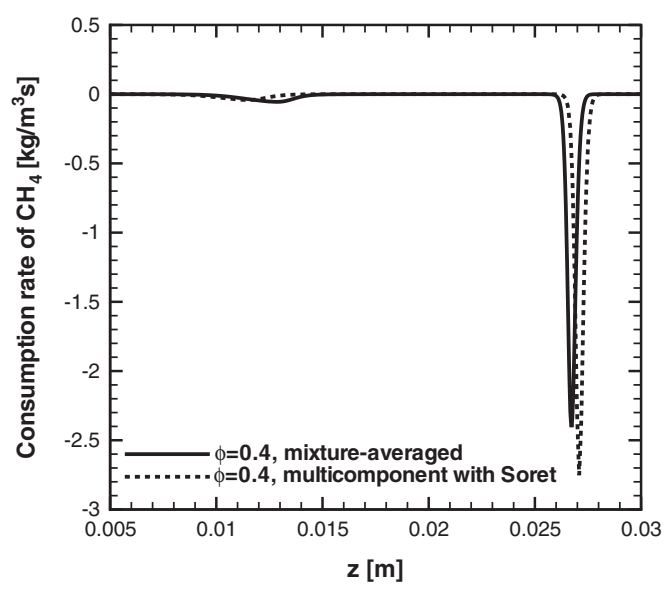

Fig. 19. Comparison of the variation of $\mathrm{CH}_{4}$ net consumption rate along the centerline for an inlet equivalence ratio $\phi=0.4$ with mixture-averaged transport, and multicomponent transport with Soret and Dufour effects included. Reaction mechanism: GRI-Mech 3.0.

around the flame front, $\mathrm{H}_{2}$ experiences an additional drift towards the flame front, which increases the $\mathrm{H}_{2}$ flux to the flame front and leads to predictions of larger flame radii and lower flammability limits. These parameters are in fact more affected by second-order transport than by the choice of the reaction mechanism, at least for the two chemical schemes under consideration. For the flame ball radius, the influence of thermal diffusion becomes more significant as the equivalence ratio is reduced. For instance, when GRI-Mech 3.0 is considered, inclusion of thermal diffusion leads to an increment in the radius of approximately $21 \%$ for $\phi=0.4$ and $69 \%$ for $\phi=0.325$. Despite these differences introduced by the inclusion of second-order transport, the radii of the flame balls would seem to become more comparable in the vicinity of the lean limit for the same reaction mechanism.

As for the normal-gravity burner-stabilized flames, an additional simulation is performed to highlight the influence of the Soret effect on the characteristics of the flame. In Fig. 18 the net consumption rates of $\mathrm{CH}_{4}$ and $\mathrm{H}_{2}$ for the premixed mixture with inlet $\phi=0.4$ are compared with and without inclusion of Soret and Dufour effects and chemistry described by the GRI-Mech 3.0. It can be observed that the inclusion of these effects intensifies both the $\mathrm{CH}_{4}$ and $\mathrm{H}_{2}$ net consumption rates. The intensification of the consumption of $\mathrm{H}_{2}$ is more localized in the upper outer region of the cap-like front. As a consequence of the intensified burning of fuel, the flame stabilizes at a higher position as compared to the flame computed without second-order diffusion processes. The standoff distance is decreased by approximately $12 \%$, as compared to that reported earlier in Section 5.2. Concerning the dimensions of the flame, the height and width are both increased by nearly $13 \%$ and $8 \%$, respectively.

The centerline variations of the consumption rates of $\mathrm{CH}_{4}$ and $\mathrm{H}_{2}$ are displayed in Figs. 19 and 20, respectively. While at the leading edge of the flame the consumption rates of $\mathrm{CH}_{4}$ and $\mathrm{H}_{2}$ are both intensified with the inclusion of thermal diffusion, at the trailing edge the situation differs. In this case, $\mathrm{H}_{2}$ exhibits a larger absolute peak rate with the inclusion of thermal diffusion, but not $\mathrm{CH}_{4}$. Also, the consumption of $\mathrm{H}_{2}$ spreads over a larger distance at the trailing edge due to enhanced diffusion by the Soret effect. Another consequence of the inclusion of second-order transport is an increase of temperature, which can be seen in Fig. 21, where the variation of temperature along the centerline is shown.

By accounting for thermal diffusion and having a more accurate representation of transport, the prediction of standoff distance and flame size would seem to worsen as compared to the measurements. 


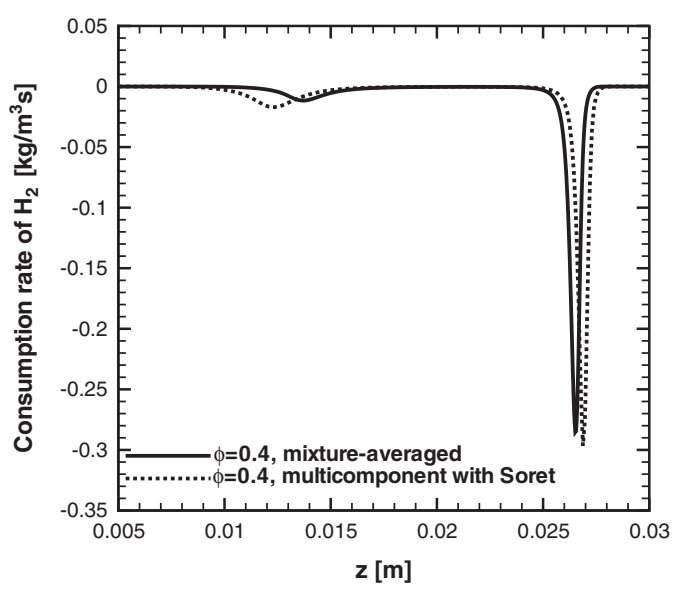

Fig. 20. Comparison of the variation of $\mathrm{H}_{2}$ net consumption rate along the centerline for an inlet equivalence ratio $\phi=0.4$ with mixture-averaged transport, and multicomponent transport with Soret and Dufour effects included. Reaction mechanism: GRI-Mech 3.0.

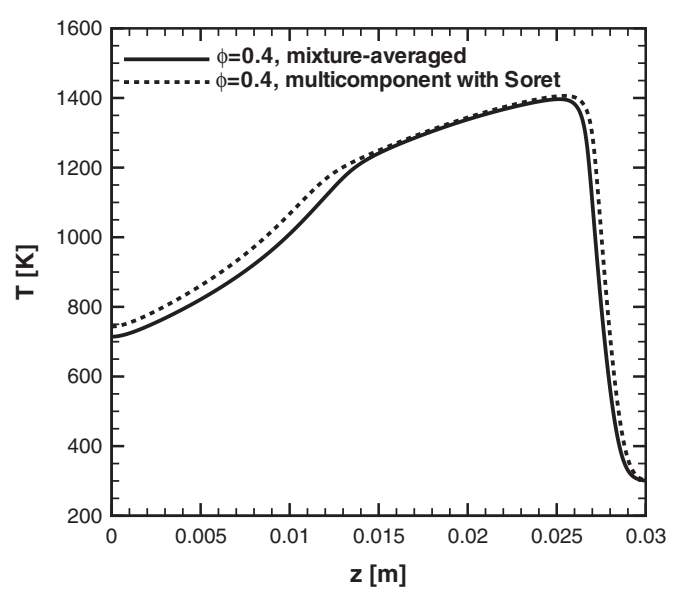

Fig. 21. Comparison of the variation of temperature along the centerline for an inlet equivalence ratio $\phi=0.4$ with mixture-averaged transport, and multicomponent transport with Soret and Dufour effects included. Reaction mechanism: GRI-Mech 3.0 .

Provided that the chemical kinetic scheme is accurate for the conditions studied, one would expect the opposite outcome. Hence, this result hints at inaccuracies in the reaction mechanism.

In essence, the inclusion of thermal diffusion leads to predictions of larger flame sizes for both the gravity-free flame balls and the burner-stabilized normal-gravity flame considered here. Also, lower lean limits are predicted when thermal diffusion is included in the computations of the gravity-free flame balls. Although the enhanced diffusion and consumption rate of $\mathrm{H}_{2}$ by the Soret effect might imply changes in the predictions of the limit flame for the burner configuration, determining the limit flame with inclusion of thermal diffusion is beyond the scope of the present study.

\section{Conclusions}

Laminar, axisymmetric, steady, and ultra-lean reacting fronts transitioning to a ball-like flame were studied at terrestrial-gravity conditions for premixed mixtures containing $40 \% \mathrm{H}_{2}$ and $60 \% \mathrm{CH}_{4}$ (on a molar basis) in the fuel. Two-dimensional simulations were performed with mixture-averaged transport and the GRI-Mech 3.0 reaction mechanism. Although the transition from a cap-like to a nearly-spherical flame was predicted in good qualitative agreement with experimental observations, the computed flames displayed a larger size and a shorter standoff distance as compared to their experimental counterparts. The influence of chemical kinetic scheme on the numerical predictions was also examined by carrying out additional simulations with a skeletal reaction mechanism, yielding predictions that compared slightly unfavorably to those of GRI-Mech 3.0.

In addition, solutions of perfectly-spherical flame balls were computed and compared with the terrestrial-gravity flames for both the two-dimensional simulations and the experiments. The peak values of the absolute molar consumption rates of both $\mathrm{H}_{2}$ and $\mathrm{CH}_{4}$ for the two-dimensional limit flame are significantly larger than those of the corresponding flame ball. To some extent, the fuel supply mechanism of the two-dimensional limit flame is similar to the fuel supply of flame balls in that it is diffusion driven even away from the flame front. The fuel flux exhibits differential diffusion, which is more pronounced for $\mathrm{H}_{2}$. In contrast to microgravity flame balls, it was found from the simulation results that the heat loss by thermal radiation is negligibly small. Instead, heat conduction to the burner tube wall and convective heat loss are the dominant form of heat loss.

Additional simulations with inclusion of multicomponent transport and Soret and Dufour effects show that the flame size increases for both flame balls and the burner-stabilized flames when second-order transport effects are incorporated in the computations. For the former case, a leaner limit is also predicted. For the latter case, a slight modification in the stabilization position is found due to the intensification of the consumption rates of both $\mathrm{H}_{2}$ and $\mathrm{CH}_{4}$. The intensification in fuel consumption is more localized for $\mathrm{H}_{2}$, which is also consumed over an extended region downstream.

Future research will be conducted on the influence of the tube dimensions, mixture composition and inflow velocity on the characteristics of normal-gravity ball-like flames and further contrasting these flames to gravity-free flame balls. Moreover, since the setup considered in this study allows for the characterization of stable and steady laminar flames at ultra-lean and near-limit conditions, thus permitting the validation of models at such conditions, the evaluation of other reaction mechanisms and further examination of the Soret effect will also be contemplated.

\section{Acknowledgments}

Financial support for this research is provided by the Dutch Technology Foundation (STW). The authors thank Prof. Clinton Groth for providing access to the CFFC (Computational Framework for Fluids and Combustion) code.

\section{References}

[1] Y. Zeldovich, Theory of Combustion and Detonation of Gases, Academy of Sciences (USSR), 1944.

[2] J. Buckmaster, S. Weeratunga, Combust. Sci. Tech. 35 (1983) 287-296.

[3] B. Dehaies, G. Joulin, Combust. Sci. Tech. 37 (1984) 99-116.

[4] P. Ronney, Combust. Flame 82 (1990) 1-14.

[5] J. Buckmaster, G. Joulin, P. Ronney, Combust. Flame 79 (1990) 381-392.

[6] J.D. Buckmaster, G. Joulin, P.D. Ronney, Combust. Flame 84 (1991) 411-422.

[7] P. Ronney, K. Whaling, A. Abbud-Madrid, J. Gatto, V. Pisowicz, AIAA J. 32 (1994) 569-577.

[8] P.D. Ronney, M.-S. Wu, H.G. Pearlman, K.J. Weiland, AIAA J. 36 (1998) 13611368.

[9] J. Buckmaster, M. Smooke, V. Giovangigli, Combust. Flame 94 (1993) 113-124.

[10] M.-S. Wu, J.-B. Liu, P.D. Ronney, Proc. Combust. Inst. 27 (1998) 2543-2550.

[11] M.-S. Wu, P.D. Ronney, R.O. Colantonio, D.M. Vanzandt, Combust. Flame 116 (1999) 387-397.

[12] Y. Shoshin, L. de Goey, Exp. Thermal Fluid Sci. 34 (2010) 373-380.

[13] Y. Shoshin, J. van Oijen, A. Sepman, L. de Goey, Proc. Combust. Inst. 33 (2011) 1211-1218. 
[14] B. Oostenrijk, Earth Gravity Flame Balls, or Are They? Master's Thesis Eindhoven University of Technology, 2012. <http://www.tue.nl/en/ publication/ep/p/d/ep-uid/279412>.

[15] J.O. Hirschfelder, C.F. Curtiss, R.B. Bird, Molecular Theory of Gases and Liquids John Wiley \& Sons, New York, 1964.

[16] C.P.T. Groth, S.A. Northrup, Paper 2005-5333, AIAA, 2005.

[17] S.A. Northrup, C.P.T. Groth, Paper 2005-0547, AIAA, 2005.

18] X. Gao, C.P.T. Groth, J. Comput. Phys. 229 (2010) 3250-3275.

[19] M.R.J. Charest, C.P.T. Groth, O.L. Gülder, Combust. Theory Modell. 14 (2010) $793-825$.

[20] X. Gao, S.A. Northrup, C.P.T. Groth, Prog. Comput. Fluid Dyn. 11 (2011) 76-95.

[21] F.E. Hernández-Pérez, F.T.C. Yuen, C.P.T. Groth, O.L. Gülder, Proc. Combust. Inst. 33 (2011) 1365-1371.

[22] M.R.J. Charest, H.I. Joo, C.P.T. Groth, O.L. Gülder, Proc. Combust. Inst. 33 (2011) 549-557.

[23] F.E. Hernández-Pérez, C.P.T. Groth, O.L. Gülder, Int. J. Hydrogen Energy 39 (2014) 7147-7157.

[24] D.G. Goodwin, Chemical Vapor Deposition XVI and EUROCVD 14 (2003) 155 162.

[25] California Institute of Technology, CANTERA Release 1.7, 2006. <http:/ code.google.com/p/cantera>.

[26] G.P. Smith, D.M. Golden, M. Frenklach, N.W. Moriarty, B. Eiteneer, M. Goldenberg, C.T. Bowman, R.K. Hanson, S. Song, W.C. Gardiner, V.V. Lissianski, Z. Qin, GRI-Mech 3.0, 2000. <http://www.me.berkeley.edu/gri_mech/>.
[27] J.-Y. Ren, W. Qin, F.N. Egolfopoulos, T.T. Tsotsis, Combust. Flame 124 (2001) $717-720$.

[28] J.-Y. Ren, W. Qin, F.N. Egolfopoulos, H. Mak, T.T. Tsotsis, Chem. Eng. Sci. 56 (2001) 1541-1549.

[29] M.D. Smooke, V. Giovangigli, in: M.D. Smooke (Ed.), Lecture Notes in Physics, vol. 384, Springer-Verlag, Berlin, 1991, pp. 1-28.

[30] A. Soufiani, J. Taine, Int. J. Heat Mass Trans. 40 (1997) 987-991.

[31] M.-S. Liou, J. Comput. Phys. 214 (2006) 137-170.

[32] V. Venkatakrishnan, Paper 93-0880, AIAA, 1993.

[33] W.J. Coirier, An Adaptively-Refined, Cartesian, Cell-Based Scheme for the Euler and Navier-Stokes Equations, Ph.D. Thesis, University of Michigan, 1994.

[34] J.M. Weiss, W.A. Smith, AIAA J. 33 (1995) 2050-2057.

[35] B. van Leer, C.H. Tai, K.G. Powell, Paper 89-1933-CP, AIAA, 1989

[36] L.M.T. Somers, The Simulation of Flat Flames with Detailed and Reduced Chemical Models, Ph.D. Thesis, Eindhoven University of Technology, 1994.

[37] J.D. Buckmaster, G. Joulin, Combust. Sci. Tech. 89 (1993) 57-69.

[38] R. Hermanns, Laminar Burning Velocities of Methane-Hydrogen-Air Mixtures, Ph.D. Thesis, Eindhoven University of Technology, 2007.

[39] N. Brown, L. Bastien, P. Price, Prog. Energy Combust. Sci. 37 (2011) 565-582.

[40] A. Sánchez, F. Williams, Prog. Energy Combust. Sci. 41 (2014) 1-55.

[41] F. Yang, C. Law, C. Sung, H. Zhang, Combust. Flame 157 (2010) 192-200.

[42] W. Liang, Z. Chen, F. Yang, H. Zhang, Proc. Combust. Inst. 34 (2013) 695-702.

[43] J.F. Grcar, J.B. Bell, M.S. Day, Proc. Combust. Inst. 32 (2009) 1173-1180.

[44] A. Ern, V. Giovangigli, Combust. Theory Modelling 2 (1998) 349-372. 